The diagnosis of AS should be considered in infants with developmental delay and generalized cryptogenic seizures resistant to medication. Diagnosis is confirmed in $85 \%$ by genetic testing and deletion of chromosome 15q11-13. In rare cases, other genetic mechanisms have been uncovered, and in $15 \%$ of cases, diagnosis is made on clinical and EEG findings alone. Where possible, the genetic cause should be determined as a prelude to family counseling.

\title{
RISK OF EMERGENCY INTRACRANIAL PATHOLOGY AFTER FIRST COMPLEX FEBRILE SEIZURE
}

The outcome of 71 neurologically normal children after a first complex febrile seizure (CFS) was determined by review of neuroimaging findings, telephone interviews, and medical records, in a prospective study at Columbia University College of Physicians and Surgeons, New York. During the study period March 1999-July 2002, 293 children presented to the ED with a first febrile seizure and of these, $79(27 \%)$ were complex in type. Complex characteristics (focal, multiple episodes in a 24 hour period, and prolonged duration $>15 \mathrm{~min}$ ) were single in $51(72 \%)$, of which 20 were focal, 22 multiple episodes, and 9 prolonged CFS. Twenty had seizures with multiple complex features. Lumbar puncture was performed in $10(14 \%)$ in the ED, and none had meningitis. Emergency cranial CT scans were obtained in the ED for 10 patients (14\%), and $13(18 \%)$ patients were admitted. A total of $46(65 \%)$ patients had emergency CT scans in the ED and/or MRI scans within 1 week after the ED visit and were followed prospectively. Outcome was determined by telephone interview within the subsequent 4 to 44 months (median 22.4 months) in 12 (17\%) patients and by medical record review in $13(18 \%)$. Of the 71 patients studied, none had intracranial pathology requiring emergency medical or neurosurgical intervention $(95 \%$ confidence interval: $4 \%$ ). It is concluded that the risk of emergency intracranial pathology following a CFS is low, and routine emergency neuroimaging in children with first complex febrile seizures is probably unnecessary. (Teng D, Dayan P, Tyler S, et al. Risk of intracranial pathologic conditions requiring emergency intervention after a first complex febrile seizure episode among children. Pediatrics February 2006;117:304-308). (Respond: Dale C Hesdorffer PhD, Gertrude H Sergievsky Center, Columbia University, $630 \mathrm{~W} 168^{\text {th }}$ St, P\&S Unit 16, New York, NY 10032).

COMMENT. In children presenting with a complex febrile seizure (CFS), each patient must be evaluated clinically to exclude possible meningitis, encephalitis or abscess, and routine neuroimaging in the ED is considered unnecessary. CT scan is not without risk, both from radiation and the need for sedation. Risk factors for subsequent epilepsy in children with FS include the CFS, a family history of epilepsy, and neurodevelopmental abnormality (in Commentary by DiMario FJ Jr. Pediatrics 2006;117:528-530); adapted from Shinnar S et al. J Child Neurol 2002;17(suppl 1):S44-S52). Earlier studies found that the duration of the FS was the most important determinant of the later development of epilepsy and an abnormal EEG (Millichap JG et al. Neurology 1960;10:643-653; Maher J et al. Brain 1995;118:1521-1528). Neuroimaging should be considered in the individual patient with a CFS that is very prolonged or is followed by a persistent epileptiform abnormality in the EEG. 
Risk factors for recurrence of febrile seizures were reported in a study of FS in Southern Chinese children (Chung BC et al. Pediatr Neurol 2006;34:121-126). Factors reaching statistical significance included: 1) earlier age of onset $<1.5$ years; 2) family history of FS; 3 ) CFS as first FS; and 4) relatively lower degree of fever (low threshold) with first FS in patients with recurrence vs those with no recurrence. Factors not significant in determining risk of recurrence of FS included sex, family history of epilepsy, and duration of fever during the FS illness. This study confirms the long established theory of height of the fever as a measure of the FS threshold and risk factor for recurrent FS and epilepsy. (See Ped Neur Briefs May 1995 and May 1994).

\section{LATERALIZING SIGNS IN PARTIAL EPILEPSY}

Lateralizing signs (LSs) in 100 children of 12 or $<12$ years of age with localizationrelated epilepsy were studied while undergoing presurgical evaluation at the Epilepsy Center Bethel, Bielefeld, Germany. Seizures were localized by video-EEG recordings and cortical resections and involved the frontal lobe in $21 \%$ patients, temporal lobe in $61 \%$, occipital lobe in $12 \%$, and parieto-occipital in $6 \%$. The side of seizure onset was defined by seizure freedom after cortical resection. Etiology of epilepsy was a cortical malformation in 49, dysgenetic tumor in 32 , hippocampal sclerosis in 9 , and various pathologies, including tuberous sclerosis, stroke, Sturge-Weber syndrome, and abscess, in 10. LSs occurred in $75 \%$ children and were more frequent in older children $(p=0.001)$. The following LSs were significantly more common in older children (manual automatism, dystonic posturing, version, postictal dysphasia, and postictal facial wiping), whereas the following showed no significant differences with age (postictal nose wiping, unilateral tonic or clonic seizures, Todd's paralysis, and nystagmus). The sum of different LSs showed a linear relation with age at monitoring $(\mathrm{p}<0.001)$. (Fogarasi A, Janszky J, Tuxhorn I. Peri-ictal lateralizing signs in children: blinded multiobserver study of 100 children $<12$ years. Neurology January ( 2 of 2$)$ 2006;66:271-274). (Reprints: Dr Andras Fogarasi, Epilepsy Center, Bethesda Children's Hospital, Bethesda Street 3, H-1146-Budapest, Hungary).

COMMENT. LSs are less frequent in children than in adults but are of high localizing value. They are more common in older than in younger children, and their development appears to correlate with brain maturation.

Partial seizures in progressive multifocal leukoencephalopathy (PML). Review of records of 89 patients (age range 14-81 years; mean age 44.8 years) with PML showed a seizure prevalence of $18 \%$, and much higher than expected in a white matter disease. Seven (44\%) had simple partial seizures, $2(12 \%)$ complex partial seizures, and $7(14 \%)$ partial with secondary generalization. MRI showed lesions adjacent to hemispheric cortex in 15 (94\%) of 16 PML patients with seizures. Eighty percent of patients with PML have HIV infection or other immunosuppressive illness. New-onset partial seizures in immunosuppressed patients should signal tests for PML (Lima MA et al. Neurology Jan (2 of 2) 2006;66:262-264). 